\title{
A Kinetic Study on Magnetic Orientation of a Liquid Crystalline Copolyester
}

\author{
Tsunehisa Kimura, ${ }^{\dagger}$ Hiroaki SATA, and Eiko Ito \\ Department of Industrial Chemistry, Faculty of Engineering, Tokyo Metropolitan University, \\ Minami-ohsawa, Hachioji, Tokyo 192-0397, Japan
}

(Received September 25, 1997)

\begin{abstract}
A kinetics of the orientation of a commercial liquid crystalline copolyester $(60$ mol\% $\%$-hydroxybenzoic acid $/ 40 \mathrm{~mol} \%$ ethylene terephthalate) in the magnetic field (6 T) was studied by means of X-ray measurements. The data were analyzed in terms of the domain model and the lattice model simulation. The rates of the magnetic orientation from an initial random state of orientation were found to be of $10^{-4}$ to $10^{-2} \mathrm{~s}^{-1}$ depending on temperatures. The comparison of the results obtained by the domain model and by the simulation suggested that the inter-domain interaction is important to explain the experimental result obtained for the reorientation process.

KEY WORDS Magnetic Orientation / Kinetic Study / Liquid Crystalline Polymer / X-Ray Analysis / Simulation /
\end{abstract}

Thermotropic liquid crystalline copolyesters ${ }^{1-3}$ exhibit excellent physical and mechanical properties when they are aligned. The orientation of these polymers is usually attained by mechanical methods including the application of shear and elongation stresses through the extrusion or molding processes, but these methods could not provide a uniform orientation, resulting in imperfect orientations, even in a skin-core structure in some cases.

A magnetic field is an alternative for attaining orientations. Due to the diamagnetic anisotropy of the backbone structure, the propensity to form mesophase, and a relatively low melt viscosity, these polymers can align under magnetic fields ${ }^{2,4-8}$ and resultant oriented structures can provide physical and mechanical properties $^{9-14}$ comparable to those exhibited by oriented structures prepared with mechanical methods. The ability of the magnetic field to penetrate through materials makes it possible to produce a uniform orientation. In addition, the control of the magnetic field is easier than the control of the mechanical forces, enabling fine designs of the orientation in final products.

Studies by means of NMR, ${ }^{6,15}$ SEM, X-ray, ${ }^{7,16}$ SQUID, ${ }^{17}$ and SANS $^{18}$ have been reported on the kinetic behavior of the magnetic orientation of liquid crystalline polymers. Also the theoretical basis ${ }^{6,15,19}$ and simulation methods ${ }^{20}$ have been reported for analyses of the magnetic orientation. A simple model, in which a liquid crystalline domain rotates in a viscous media by the action of magnetic torque, has been used to describe the magnetic orientation. This model applies appropriately to the magnetic orientation of poly-domain systems with the initial random distribution, but it does not work very well in the case of reorientation process of mono-domain systems especially when the magnetic field is applied perpendicular to the original orientation direction. ${ }^{15,17,21}$

In this paper, we report the X-ray measurements on the magnetic orientation of a commercially available thermotropic liquid crystalline polymer. A model calculation and a simulation are used to analyze the experimental data, and the advantage and the limitations of these analyzing methods are examined for the future work in developing the processing methods of liquid crystalline polymers by means of magnetic fields.

\section{EXPERIMENTAL}

\section{Sample Preparation}

A Unitika liquid crystalline copolyester, Rodrun LC3000 , consisting of $60 \mathrm{~mol} \%$ p-hydroxybenzoic acid/ $40 \mathrm{~mol} \%$ ethylene terephthalate (Figure 1) was dried at $80^{\circ} \mathrm{C}$ for $24 \mathrm{~h}$ under the vacuum, melt at $230^{\circ} \mathrm{C}$ followed by hot press at $230^{\circ} \mathrm{C}$ for $20 \mathrm{~s}$, and quenched in ice water. The melt of this material exhibited a liquid crystalline phase over the temperatures studied in this work. The quenched samples were of liquid crystalline glass. The film obtained was dried for $24 \mathrm{~h}$ under the vacuum and cut into small pieces of films of $20 \mathrm{~mm} \times$ $30 \mathrm{~mm}$ in size. These films were subjected to the X-ray analysis to check the residual orientation caused during the hot pressing and those exhibiting little orientation were chosen for the further heat treatment in the magnet.

\section{Heat Treatment in the Magnet}

The film specimen was heat treated in the magnet $(6 \mathrm{~T})$ by using the apparatus shown in Figure 2 . The tem-

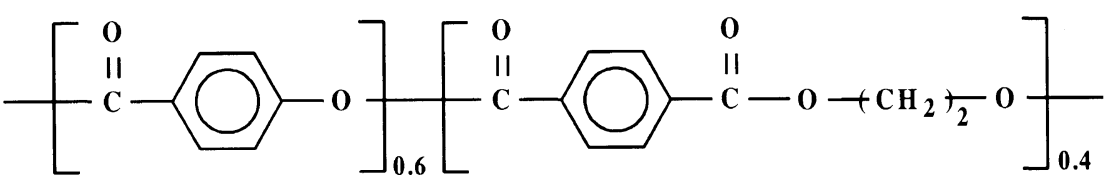

Figure 1. Chemical structure of the liquid crystalline random copolyester used in this study.

\footnotetext{
† To whom correspondence should be addressed.
} 
perature was controlled by PID controller and the error in temperature was $\pm 1^{\circ} \mathrm{C}$. The specimen was set in the magnet and heated at $10^{\circ} \mathrm{C} \mathrm{min}^{-1}$ and brought to the heat treatment temperature ranging from 230 to $255^{\circ} \mathrm{C}$, followed by the isothermal treatment for 0 to $20 \mathrm{~h}$. After the heat treatment, the specimen was removed from the magnet and quenched in ice water. Because there was no difference in orientation degree between the sample quenched outside the magnet and the sample prepared by slowly cooling in the magnet, the orientation of quenched samples could represent the orientation which the samples are assumed to have in molten states in the magnet. For the experiment of reorientation, some of the oriented specimens thus obtained was heat treated again in the same manner as for the first treatment with the orientation direction of the specimen being set perpendicular to the magnetic field.

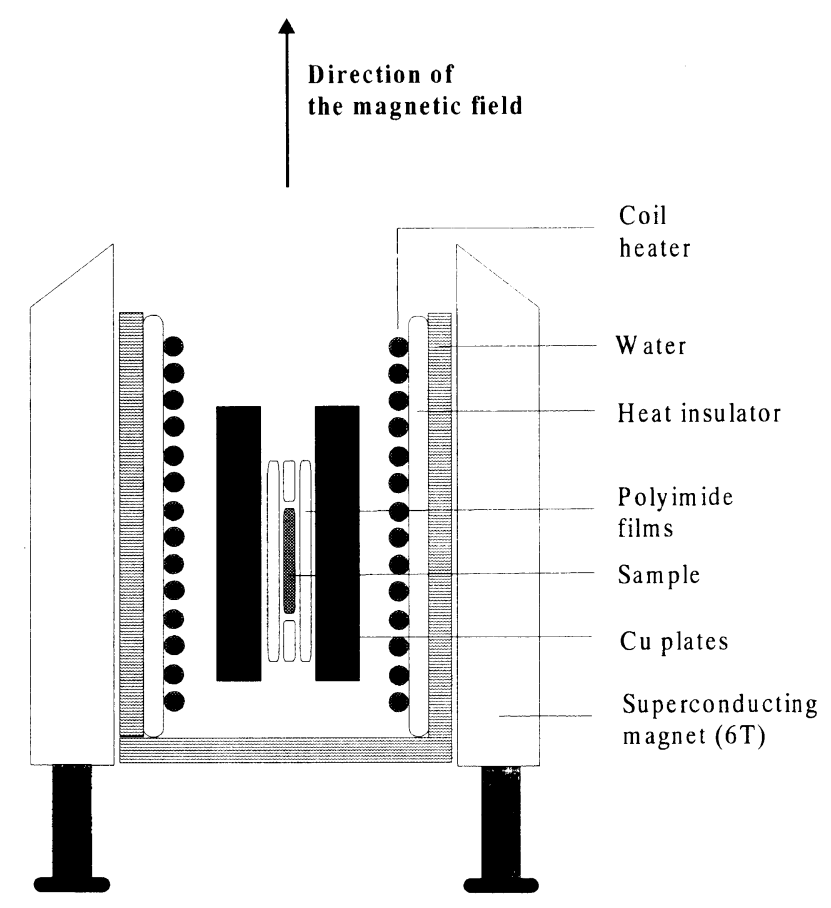

Figure 2. The apparatus used for the preparation of film samples in a superconducting magnet $(6 \mathrm{~T})$.

\section{$X$-Ray Measurement}

$\mathrm{X}$-Ray measurements were carried out on a MAC Science MXP18 using a Ni-filtered $\mathrm{Cu}-K_{\alpha}$ X-ray beam from a rotating-anode $\mathrm{X}$-ray generator operating at $40 \mathrm{kV}$ and $250 \mathrm{~mA}$. Azimuthal scans were carried out at $2 \Theta=20.0^{\circ}$ with the scan rate of $60^{\circ} \mathrm{min}^{-1}$.

\section{Melt Viscosity Measurement}

Melt viscosity was measured by using a Rheometrics RDA-II. The sample was melted at $270^{\circ} \mathrm{C}$ and cooled to the measurement temperature. Measurements were carried out every $5^{\circ} \mathrm{C}$ down to $220^{\circ} \mathrm{C}$. Similar measurements were carried out by heating the sample from $220^{\circ} \mathrm{C}$. The shear rate was $0.1 \mathrm{rad} \mathrm{s}^{-1}$.

\section{MODELING AND SIMULATION}

\section{Domain Model}

Following Moore and Stupp, ${ }^{6}$ we consider a 'domain model.' We assume that a director $\boldsymbol{n}$, representing a spherical nematic domain immersed in the surrounding viscous media of the same material, rotates under the torque exerted by an external magnetic field. The equation of motion of the domain is given by the balance of the magnetic torque and the viscous resistance, and is written as

$$
\eta \frac{\mathrm{d} \theta}{\mathrm{d} t}+\chi_{\mathrm{a}} \mu_{0} H^{2} \sin \theta \cos \theta=0
$$

where $\eta, \mu_{0}$, and $H$ are the viscosity, the magnetic permitivity of the vacuum, and the strength of the magnetic field, respectively, $\chi_{\mathrm{a}}$ represents the diamagnetic anisotropy $\chi_{\|}-\chi_{\perp}$, and $\theta$ is the angle between the magnetic field $\boldsymbol{H}$ (the direction of the $z$-axis) and the director $\boldsymbol{n}$ (Figure 3a). The solution of eq 1 is given as

$$
\tan \theta=e^{-t / \tau} \tan \theta_{0}
$$

where the $\theta_{0}$ is the initial angle and $\tau$ is defined as

$$
\tau=\frac{\eta}{\chi_{\mathrm{a}} \mu_{0} H^{2}}
$$

During the rotation, the angle $\varphi_{0}$ remains unchanged.

Equation 2 is for the motion of a single domain. This is easily extended to the case with a multi-domain system (a)

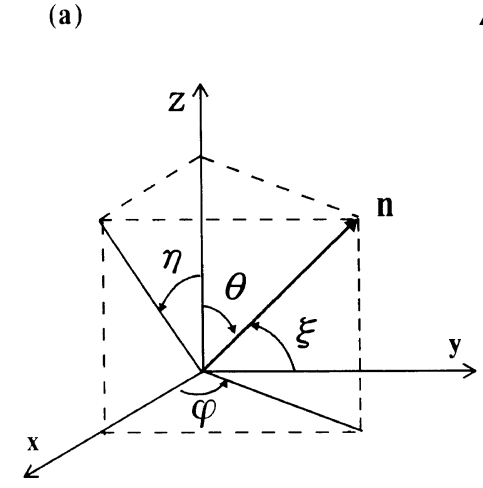

H (First orientation)

(reorientation) (b)

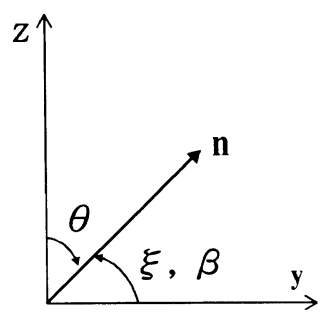

Figure 3. Polar coordinates defining the direction of the director $\boldsymbol{n}$ in the three dimensions (a) and in the two dimensions (b). The magnetic field is first applied to the $z$-direction for the period of $t_{1}$, followed by a subsequent application to the $y$-direction (reorientation) for the period of $t_{2}$. The angle $\beta$ indicates the azimuthal angle in the X-ray measurement. 
if the interaction between domains is neglected. ${ }^{6}$ The initial distribution $P_{0}\left(\theta_{0}, \varphi_{0}\right)$ of domains changes to the distribution $P_{1}\left(\theta, \varphi_{0}\right)$ after the time $t_{1}$ during which the individual domains change their initial angles independently, according to eq 2 . In the case of the initial random orientation, $P_{0}$ is put as $1 / 4 \pi$, resulting in $P_{1}$ same as derived by Moore and Stupp. ${ }^{6}$ Then, we switch the direction of the magnetic field from the $z$-axis to the $y$-axis and apply the magnetic field for the period of $t_{2}$ (reorientation). Since it is convenient to use the variables $(\xi, \eta)$ (Figure $3 a)$ to describe the reorientation toward the $y$-axis, the distribution $P_{1}\left(\theta, \varphi_{0}\right)$ is then rewritten in terms of $\left(\xi_{0}, \eta_{0}\right)$, that is, $Q_{1}\left(\xi_{0}, \eta_{0}\right)=P_{1}\left(\theta\left(\xi_{0}, \eta_{0}\right)\right.$, $\left.\varphi_{0}\left(\xi_{0}, \eta_{0}\right)\right)$. The subscript 0 on $\xi$ and $\eta$ indicates that $\left(\xi_{0}, \eta_{0}\right)$ is the initial angle before the reorientation. The temporal change of the angle $\xi_{0}$ is given by the equation similar to eq 2 , resulting in a new distribution $Q_{2}\left(\xi, \eta_{0}\right)$. The detail of the derivation of $P_{1}\left(\theta, \varphi_{0}\right)$ and $Q_{2}\left(\xi, \eta_{0}\right)$ is given in the appendix.

\section{Simulation}

A simulation of the magnetic orientation was carried out as follows: The two dimensional lattice model simulation $^{20}$ is employed and applied to the simulation of reorientation. In addition to the term of the magnetic torque in eq 1 , this model includes the interaction between directors through the Lebwohl-Lasher potential, ${ }^{22}$ and the effect of thermal agitation through Langevin force. ${ }^{23}$ It is possible to include the asymmetry between the bending and splay elastic constants in order to incorporate the polymeric effect on the interaction with the neighboring directors ${ }^{24}$ but it is not included here. The equation of motion is written as

$$
\frac{\Delta \theta_{i j}}{\Delta t}=\frac{1}{\tau_{0}} \sum_{N N} \sin 2\left(\theta_{N N}-\theta_{i j}\right)-\frac{1}{2 \tau} \sin 2 \theta_{i j}+\sqrt{D} g_{i j}(t) / \Delta t
$$

where $\theta_{i j}$ is the angle of the director located at the lattice site $(i j)$ measured against the direction of the magnetic field, $\theta_{N N}$ is the director angles of the four neighboring sites, $\tau_{0}=K / \eta$ with $K$ being the elastic constant, $D$ is the rotational diffusion constant, and $\tau$ is given by eq 3. The function $g_{i j}$ represents the Langevin force, which satisfies

$$
\begin{aligned}
& \left\langle g_{i j}(t)\right\rangle=0 \\
& \left\langle\left(g_{i j}(t) g_{k l}\left(t^{\prime}\right)\right\rangle=2 \Delta t \delta_{i j, k l} \delta\left(t-t^{\prime}\right)\right.
\end{aligned}
$$

and gives the following average in the absence of the magnetic field and the interaction with the neighboring sites:

$$
\begin{aligned}
& \left\langle\Delta \theta_{i j}\right)=0 \\
& \left\langle\left(\Delta \theta_{i j}\right)^{2}\right\rangle=2 D \Delta t
\end{aligned}
$$

Equation 4 is numerically integrated by use of RungeKutta method.

\section{RESULTS AND DISCUSSION}

Figure 4 shows the azimuthal scans of a pressed film viewed from different directions with respect to the film surface. The scans are carried out at $2 \Theta=20.0^{\circ}$ which

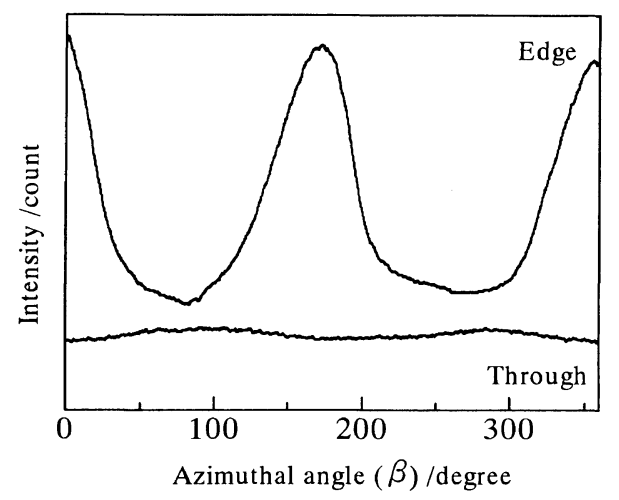

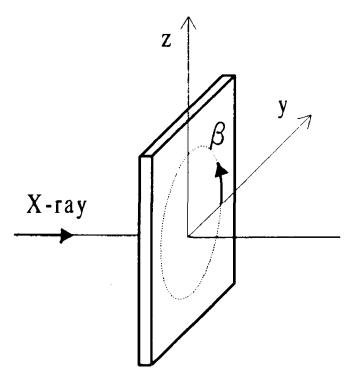

Through
view

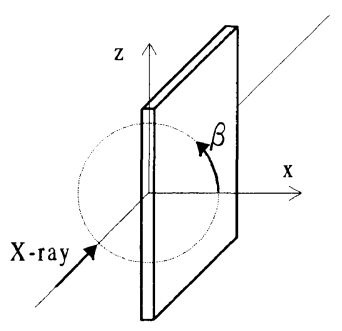

Edge

view
Figure 4. X-Ray azimuthal scans at $2 \Theta=20.0^{\circ}$ for the pressed film carried out on the edge-view and the through-view. Zero azimuthal angle for the edge-view is the direction perpendicular to the film surface. The edge-view profile indicates that the polymer chains run parallel to the film surface, while the through-view indicates that there is no orientation within the film surface.

corresponds to the spacing between chains in the nematic phase. $^{25,26}$ The edge view exhibits peaks at 0 and $180^{\circ}$, which indicates that the chain axes lie preferentially parallel to the film surface (planar orientation), while the through view exhibits no peaks, which indicates that the chain axes are uniformly distributed within the plane. This observation allows us to analyze the phenomenon under study as occurring in the two dimensions.

In the two dimensions, the distribution functions for the direction of the domain are reduced to be of simple form because the director $\boldsymbol{n}$ is on the $y-z$ plane. In addition, the distribution could be directly compared with the X-ray azimuthal distribution: The distribution $Q(\xi)$ is proportional to the $\mathrm{X}$-ray azimuthal profile $I(\beta)$. It should be noted that in comparing the model or simulation results with the $\mathrm{X}$-ray azimuthal measurements, the azimuthal angle $\beta$ is different by $\pi / 2$ with respect to $\xi$ because the direction of $\boldsymbol{n}$ represents the chain axis, while in the X-ray measurements the direction of the chain spacing is perpendicular to the chain axis. We start with a random orientation of $n$ on the $y-z$ plane (Figure 3b), followed by the application of the magnetic field in the $z$-direction for the duration of $t_{1}$ and the subsequent application in the $y$-direction (reorientation) for the duration of $t_{2}$. The resultant distribution is expressed as follows: 


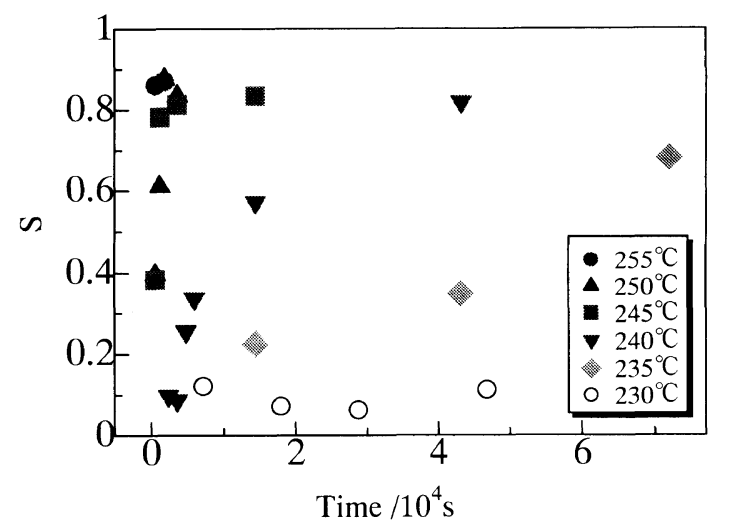

Figure 5. Temporal change of the order parameter evaluated by the $\mathrm{X}$-ray azimuthal distribution as a function of the elapsed time in the magnet.

$$
\begin{aligned}
Q_{2}(\xi)= & \frac{\mathrm{e}^{\left(t_{1}+t_{2}\right) / \tau} \csc ^{2}\left(\tan ^{-1}\left(\mathrm{e}^{t_{2} / \tau} \tan \xi\right)\right)}{2 \pi\left(1+\mathrm{e}^{2\left(t_{1}-t_{2}\right) / \tau} \cot ^{2} \xi\right)} \\
& \cdot \frac{\sec ^{2} \xi}{\left(1+\mathrm{e}^{2 t_{2} / \tau} \tan ^{2} \xi\right)}
\end{aligned}
$$

Figure 5 shows the change in order parameter $S$ as a function of the residence time during the first orientation process. Under the assumption of the two-dimensional (planar) orientation, $S$ is evaluated by the X-ray azimuthal distribution $I(\beta)$ as follows:

$$
S=2 \int_{0}^{\pi} I(\beta) \cos ^{2} \beta \mathrm{d} \beta / \int_{0}^{\pi} I(\beta) \mathrm{d} \beta-1
$$

The maximum attainable order parameter, $S_{\max }$, does not reach the unity, but it seems to take the same value for the measurements at temperatures higher than $c a$. $240^{\circ} \mathrm{C}$. The value of $S_{\max }$ is lower for the measurements at lower temperatures, and even no orientation is observed at $230^{\circ} \mathrm{C}$. The initial pressed film could possess structure inhomogeneities ${ }^{11,27}$ which cause a melting point distribution. Regions with high melting points could thus resist the orientation, resulting in a smaller $S_{\max }$ at lower temperatures. There may be disordered regions which may not contribute to the orientation but just increase the background in the azimuthal profile. Also, disclination points unavoidably associated with the multi-domain structure could be annihilated during the heat-treatment in the magnet, which might affect $S_{\max }$ value. The degree of the annihilation however may depend on the temperature. In addition, the local order parameter within a domain could depend on the temperature and the thermal history. ${ }^{11}$ All these factors could cause the temperature dependence and non-unity value of $S_{\max }$.

In the domain model, the temporal change of the distribution $P_{1}(\theta)$ during the first orientation is given by putting $t_{2}=0$ and $\xi=\pi / 2+\theta$ in $Q_{2}$ given in eq 7 :

$$
P_{1}(\theta)=\frac{\mathrm{e}^{t_{1} / \tau} \sec ^{2} \theta}{2 \pi\left(1+\mathrm{e}^{2 t_{1} / \tau} \tan ^{2} \theta\right)}
$$

Using this distribution, we obtain the temporal change of the order parameter $S$ as follows:

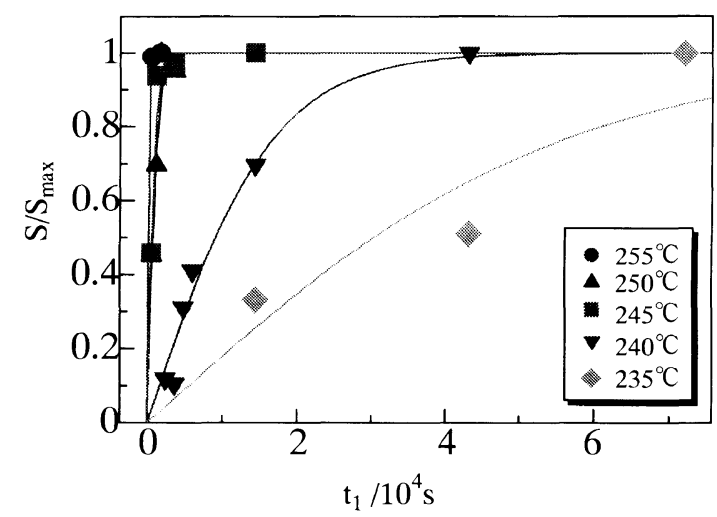

Figure 6. Order parameter normalized by $S_{\max }$. Curves are the results of fitting by the domain model with the appropriate values of $\tau$, which are summarized in Table I.

Table I. Built-up time $\tau$ required for the orientation determined by two different methods

\begin{tabular}{lccccc}
\hline & \multicolumn{5}{c}{$\tau / \mathrm{s}$} \\
\cline { 2 - 6 } & $235^{\circ} \mathrm{C}$ & $240^{\circ} \mathrm{C}$ & $245^{\circ} \mathrm{C}$ & $250^{\circ} \mathrm{C}$ & $255^{\circ} \mathrm{C}$ \\
\hline Method I & $2.76 \times 10^{4}$ & $8.26 \times 10^{3}$ & $4.95 \times 10^{2}$ & $6.10 \times 10^{2}$ & $1.16 \times 10^{2}$ \\
Method II & $2.76 \times 10^{4}$ & $7.19 \times 10^{3}$ & $1.00 \times 10^{3}$ & $9.17 \times 10^{2}$ & $7.87 \times 10^{2}$
\end{tabular}

Method I, fitting to eq 10; Method II, fitting to X-ray azimuthal profile.

$$
\begin{aligned}
S & =2 \int_{0}^{2 \pi} \cos ^{2} \theta P_{1}(\theta) \mathrm{d} \theta-1 \\
& =\tanh \left(t_{1} / 2 \tau\right) .
\end{aligned}
$$

Here, the maximum attainable order parameter $S_{\max }$ is unity when $t_{1} \rightarrow \infty$, thus the domain model is limited in applying to the case in which $S_{\max }$ is not unity. This is the case in our experiment. Nevertheless, the domain model could be used for an approximate estimation of the rate of orientation $\tau^{-1}$ by fitting the $S$ obtained by the model to the $S / S_{\max }$ plot of the X-ray data. Plots of $S / S_{\max }$ are shown in Figure 6 as a function of $t_{1}$, where each solid line in the figure represents the $S$ for the model with an appropriate $\tau$. Fitting seems fairly good. The values of $\tau$ are summarized in Table I.

Another way to estimate $\tau$ appearing in the domain model is to compare directly the $\mathrm{X}$-ray azimuthal distribution $I(\beta)$ with the distribution $P_{1}(\theta)$. Fitting is carried out by minimizing $\left(I(\beta)-a P_{1}(\theta)-b\right)^{2}$ with respect to $a, b$, and $t_{1} / \tau$ in $P_{1}(\theta)$ as fitting parameters. Figure 7 displays an example of fitting. The $\tau$ value is determined by the initial slope in the plot of $t_{1} / \tau$ versus $t_{1}$, as is shown in Figure 8. At large $t_{1}$, the point deviates largely from the initial slope. This is because the half width predicted by the domain model decreases down to zero when $t_{1} \rightarrow \infty$, while the experimental half width reaches a limiting value. This situation is similar to the situation that $S_{\max }$ does not reach the unity in the experiment. The $\tau$ values for the different temperatures determined by this method are summarized in Table I along with those determined based on $S / S_{\max }$. A good agreement is obtained over the temperature range studied except for the highest temperature, where the correct estimation of $\tau$ is difficult because of a rapid built-up 


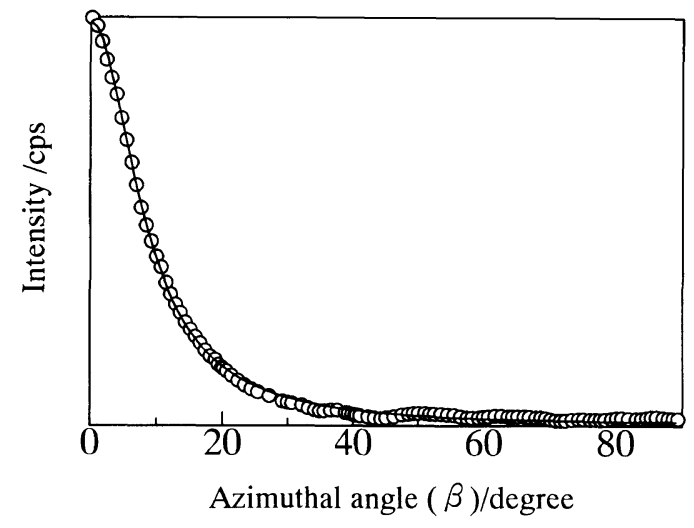

Figure 7. Profile of the azimuthal scan carried out for the sample heat treated at $255^{\circ} \mathrm{C}$ for $30 \mathrm{~min}$. Solid line indicates the fitting to the domain model.

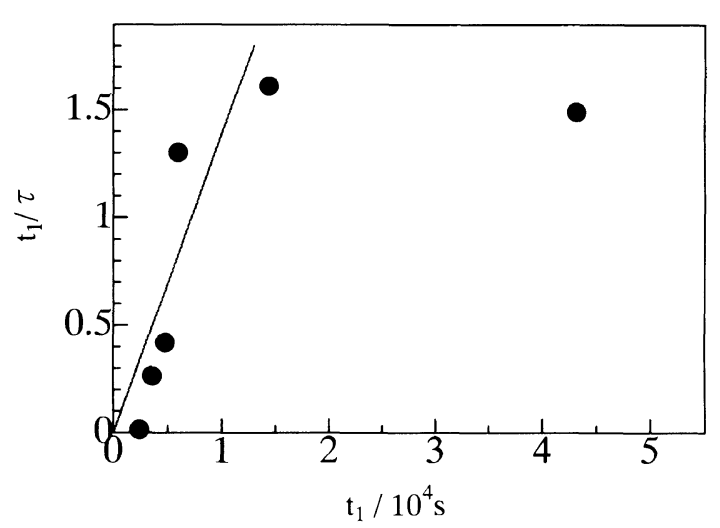

Figure 8. The values of $t_{1} / \tau$ determined by the fitting to the azimuthal profile are plotted as a function of $t_{1}$. The initial slope gives the inverse of $\tau$, which is summarized in Table $\mathbf{I}$.

\section{of the orientation.}

As indicated by eq 3, the built-up time $\tau$ necessary for the magnetic orientation is proportional to the viscosity $\eta$ of the surrounding media in the case of the domain model. The viscosity of the material used in this study shows different temperature dependence as shown in Figure 9 depending on cooling or heating. ${ }^{28}$ Since the heat-treatments in the magnetic field are carried out by a heating of the sample up to a given temperature not by a supercooling from a higher temperature, we employ the viscosity data measured at heating process. The Arrhenius plot of the viscosity $\eta$ is shown in Figure 10. In the temperature range covered in this study, the viscosity data seem to fall on a straight line. A similar observation is also reported for the same material. ${ }^{28} \mathrm{By}$ putting $H=60000 \mathrm{Oe}$ (corresponding to $6 \mathrm{~T}$ ), $\mu_{0}=1$, and $10^{-7} \mathrm{cgs}$ emu as a typical value for $\chi_{\mathrm{a}}$, the viscosity in eq 3 is expressed to be $36 \tau \mathrm{Pa} \cdot \mathrm{s}$. In Figure 10 , the viscosity thus evaluated is compared with the viscosity obtained by viscometry. Though the data are scattered, the slope seems almost similar to that obtained by viscometry, suggesting that the activation energy required for the magnetic rotation is close to that required for the flow process. On the other hand, the magnitude is higher by $1-2$ orders. A similar observation is also reported. ${ }^{6}$

Though in the domain model the interdomain interaction is neglected, and the complicated features of structure change probably occurring during the heattreatment in the magnet are not considered explicitly,

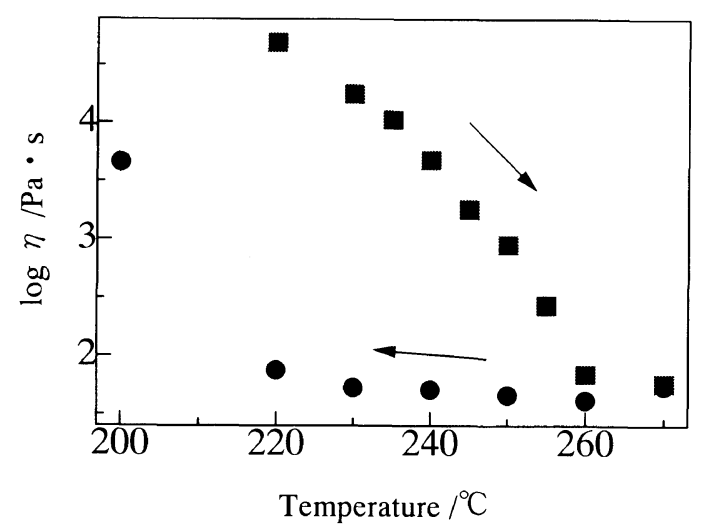

Figure 9. Viscosity measured on heating and cooling processes.

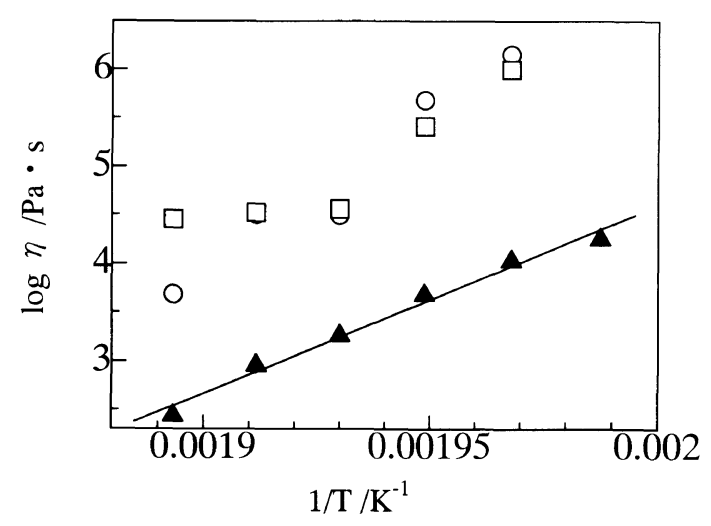

Figure 10. Arrhenius plots of the viscosity $\eta$ measured by viscometry (filled symbol) and that estimated by eq 3 (open symbols) by using $\tau$ values.

the model seems to work at least qualitatively. However, it fails when applied to a reorientation process. The reorientation here means that the domains aligned under the magnetic field are subjected to the orientation under the magnetic field applied perpendicular to the direction of the original magnetic field (Figure 3). In the case of the reorientation in the two dimensions, the individual domains exactly trace their orientation history backward to the past because the switch of the direction of the magnetic field by $\pi / 2$, i.e., replacing $\theta$ in eq 1 with $\pi / 2-\theta$, causes the minus sign in the equation of motion given in eq 1, resulting in the motion backward to the past. A typical behavior of the reorientation in two dimensions predicted by the domain model is shown in Figure 11a. Starting with the initial random orientation, the domains rotate under the effect of magnetic field applied to the $z$-direction during a period of $t_{1}$, giving peaks at $\beta=0$ and $180^{\circ}$. Then, the direction of the magnetic field is switched by $\pi / 2$, i.e., to the direction of the $y$-axis. The profile of the azimuthal distribution goes back to the past exactly the same way as it developed, and after time $t_{2}=t_{1}$, it reaches exactly the initial random distribution. On the further application of the magnetic field in the $y$-direction, the peaks come to appear at -90 and $90^{\circ}$. The above is the prediction by the domain model on the temporal change of the azimuthal profile in the course of a reorientation experiment. However, the appearance of a random state prior to the rebuilt-up of a new orientation seems physically implausible.

The experimental result of the reorientation process 

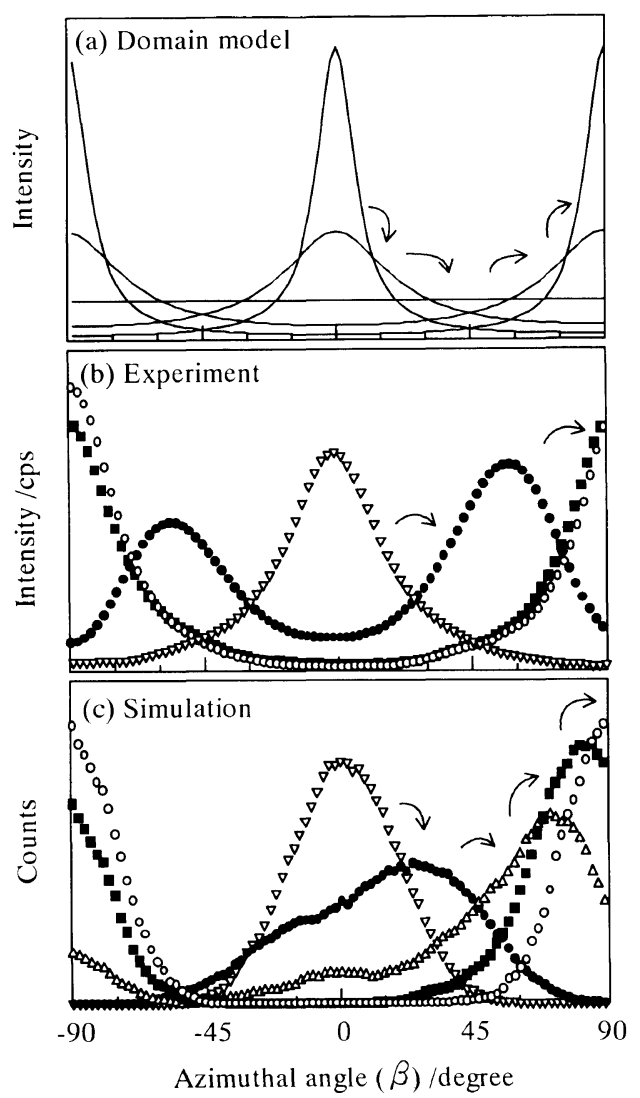

Figure 11. Temporal changes in the azimuthal profile during the reorientation measurement: the prediction by the domain model (a), the experimental result (b), and the result of the simulation (c) $\left(\tau_{0}=1\right.$, $\tau=10, D=0.1$, and $\Delta t=0.01$ in eq 4 ).

shown in Figure $11 \mathrm{~b}$ clearly demonstrates that the domain model fails to explain the reorientation behavior. In marked contrast to the prediction by the domain model, the experiment does not exhibit a flat azimuthal profile during the reorientation. Instead, the peaks initially located at 0 and $180^{\circ}$ move towards -90 or $90^{\circ}$ without disappearing during the reorientation process. The failure of equations similar to eq 1 has been reported when applied to the reorientation process especially with the reorientation angle close to $90^{\circ}$ with respect to the first orientation direction, which is the case of our present study: Temporal changes of the order parameter deviate ${ }^{15,17}$ from the behavior predicted by the equations like eq 1 . This situation is improved by the use of the equation which, in addition to the terms in eq 1 , includes the elastic term and the term coupling with the velocity (backflow). In addition, the appearance of inversion walls during the reorientation process is predicted in the case that the elastic term is included. ${ }^{21}$ The evolution of the azimuthal profile that we observe in Figure $11 \mathrm{~b}$ might be a reflection of the formation of patterns including inversion walls, though the further experimental and theoretical studies are needed to confirm this.

We finally show by using the two-dimensional simulation that the inclusion of the interaction between the adjacent domains qualitatively explains the reorientation behavior. As described in the previous section, the simulation includes the adjacent interactions as well as the Langevin force and the magnetic torque. The torque acting on $\theta_{i j}$, that is $\sin 2\left(\theta_{N N}-\theta_{i j}\right)$ in eq 4 is due to the interaction energy $\sin ^{2}\left(\theta_{N N}-\theta_{i}{ }^{j}\right)$ which corresponds to the elastic energy, $(\nabla \theta)^{2}$ in the continuum limit. ${ }^{29}$ Hence the simulation includes the elastic term. Figure $11 \mathrm{c}$ shows a simulation result of the reorientation. A characteristic feature of the temporal change in the azimuthal profile observed in the experiment is reproduced. Simulations carried out with different sets of parameters exhibited a similar tendency.

\section{CONCLUSIONS}

A kinetic study on the magnetic orientation of a liquid crystalline polymer has been reported. The development of the orientation measured by the X-ray technique was analyzed in terms of the domain model. Though the model does not include the effect of the inter-domain interactions, the magnetic orientation starting from an initial random state of domain orientation was well described by this model and the built-up rate $\tau^{1}$ of the orientation were successfully estimated. The model however failed to explain the reorientation process. The simulation on the two-dimensional lattice suggested that the failure of the domain model is attributed to its lack in the inter-domain interactions. The rates of the orientation were of the order of $10^{-4}$ to $10^{-2} \mathrm{~s}^{-1}$ under the magnetic field of $6 \mathrm{~T}$, and probably would be shorter at higher temperatures, suggesting a possibility of the actual application of magnetic fields for the processing of liquid crystalline polymers.

Acknowledgment. We thank to Rheometric Scientific F. E. Ltd. for the measurement of viscosity.

\section{APPENDIX}

An equation similar to the eq $4 \mathrm{~A}$ given below has been derived by Moore and Stupp. ${ }^{6}$ In this appendix, their equation is modified in order to describe the general cases including the reorientation in the two dimensions and the three dimensions.

\section{The First Orientation in $3 D$}

Let us assume the initial distribution function to be $P_{0}\left(\theta_{0}, \varphi_{0}\right)$. The probability of finding the director direction between $\theta_{0}$ and $\theta_{0}+\mathrm{d} \theta_{0}$, and between $\varphi_{0}$ and $\varphi_{0}+\mathrm{d} \varphi_{0}$ is written as $P_{0}\left(\theta_{0}, \varphi_{0}\right) \sin \theta_{0} \mathrm{~d} \theta_{0} \mathrm{~d} \varphi_{0}$. By the transformation given in eq 2 , the direction $\left(\theta_{0}, \varphi_{0}\right)$ moves to $\left(\theta, \varphi_{0}\right)$. The probability of finding the director direction between $\theta$ and $\theta+\mathrm{d} \theta$, and between $\varphi_{0}$ and $\varphi_{0}+\mathrm{d} \varphi_{0}$ is then written as $P_{1}\left(\theta, \varphi_{0}\right) \sin \theta \mathrm{d} \theta \mathrm{d} \varphi_{0}$. Equating these two probabilities, we obtain

$$
P_{1}\left(\theta, \varphi_{0}\right)=\frac{\sin \theta_{0}}{\sin \theta} \cdot \frac{\mathrm{d} \theta_{0}}{\mathrm{~d} \theta} \cdot P_{0}\left(\theta_{0}, \varphi_{0}\right)
$$

Substituting $\theta_{0}$ with the inverse of eq 2 :

$$
\theta_{0}=\tan ^{-1}\left(\mathrm{e}^{t_{1} / \tau} \tan \theta\right)
$$

and its derivative $\mathrm{d} \theta_{0} / \mathrm{d} \theta$ with

$$
\frac{\mathrm{d} \theta_{0}}{\mathrm{~d} \theta}=\frac{\mathrm{e}^{t_{1} / \tau}}{\cos ^{2} \theta\left(1+\mathrm{e}^{2 t_{1} / \tau} \tan ^{2} \theta\right)}
$$


we obtain $P_{1}\left(\theta, \varphi_{0}\right)$ as a function of $\theta$. For the initial random orientation, $P_{0}=1 / 4 \pi$, then we obtain

$P_{1}\left(\theta, \varphi_{0}\right)=\frac{\mathrm{e}^{t_{1} / \tau} \csc \theta \sec ^{2} \theta \sin \left(\tan ^{-1}\left(\mathrm{e}^{t_{1} / \tau} \tan \theta\right)\right)}{4 \pi\left(1+\mathrm{e}^{2 t_{1} / \tau} \tan ^{2} \theta\right)}$

This is the same as derived by Stupp and Moore. ${ }^{6}$

\section{Change of Variables in $3 D$}

We consider the change of variables from $(\theta, \varphi)$ to $(\xi, \eta)$, which is necessary to describe the reorientation process. Figure 3 a leads to the following relations:

$$
\begin{aligned}
& x=\sin \theta \cos \varphi=\sin \xi \sin \eta \\
& y=\sin \theta \sin \varphi=\cos \xi \\
& z=\cos \theta=\sin \xi \cos \eta
\end{aligned}
$$

From these equations we obtain:

$$
\begin{aligned}
& \xi=\cos ^{-1}(\sin \theta \sin \varphi) \\
& \eta=\tan ^{-1}(\tan \theta \cos \varphi)
\end{aligned}
$$

and the inverse relations

$$
\begin{aligned}
& \theta=\cos ^{-1}(\sin \xi \cos \eta) \\
& \varphi=\tan ^{-1}(\cot \xi \csc \eta)
\end{aligned}
$$

The distribution function $Q_{1}(\xi, \eta)$ in the $(\xi, \eta)$ coordinate is related to the distribution $P_{1}(\theta, \varphi)$ by the following relation:

$$
Q_{1}(\xi, \eta) \sin \xi \mathrm{d} \xi \mathrm{d} \eta=P_{1}(\theta, \varphi) \sin \theta \mathrm{d} \theta \mathrm{d} \varphi
$$

then $Q_{1}(\xi, \eta)$ is calculated as

$$
\begin{aligned}
Q_{1}(\xi, \eta) & =\frac{\mathrm{d} \theta \mathrm{d} \varphi}{\mathrm{d} \xi \mathrm{d} \eta} \frac{\sin \theta}{\sin \xi} P_{1}(\theta, \varphi) \\
& =\left|\frac{\partial(\theta, \varphi)}{\partial(\xi, \eta)}\right| \frac{\sin \theta}{\sin \xi} P_{1}(\theta, \varphi)
\end{aligned}
$$

where $\theta$ and $\varphi$ should be replaced with the inverse functions defined in eq 7A, and Jacobian is given as

$$
\frac{\partial(\xi, \eta)}{\partial(\theta, \varphi)}=\frac{\cos \xi \cot ^{2} \eta \cot \xi+\csc \xi}{\left(1+\cot ^{2} \xi \csc ^{2} \eta\right) \sqrt{1-\cos ^{2} \eta \sin ^{2} \xi}}
$$

Substituting eq $10 \mathrm{~A}$ into eq $9 \mathrm{~A}$, we obtain

$$
Q_{1}(\xi, \eta)=P_{1}(\theta, \varphi)
$$

with $\theta$ and $\varphi$ being given in eq $7 \mathrm{~A}$.

\section{Reorientation in $3 D$}

We describe the orientation of the director in the beginning of the reorientation by $\left(\xi_{0}, \eta_{0}\right)$, which corresponds to $\left(\theta, \varphi_{0}\right)$. Then, the initial distribution is given by

$$
Q_{1}\left(\xi_{0}, \eta_{0}\right)=P_{1}\left(\theta\left(\xi_{0}, \eta_{0}\right), \varphi_{0}\left(\xi_{0}, \eta_{0}\right)\right)
$$

where the function form $P_{1}$ is given in eq $4 \mathrm{~A}$. We then apply the magnetic field to the $y$-axis. The angle $\xi$ between the director and the magnetic field after the period of $t_{2}$ is given by the equation of motion

$$
\xi=\tan ^{-1}\left(\mathrm{e}^{-t_{2} / \tau} \tan \xi_{0}\right),
$$

while $\eta_{0}$ does not change. Then, the distribution function
$Q_{2}\left(\xi, \eta_{0}\right)$ after the period of $t_{2}$ should be given as

$$
Q_{2}\left(\xi, \eta_{0}\right)=\frac{\sin \xi_{0}}{\sin \xi} \frac{\mathrm{d} \xi_{0}}{\mathrm{~d} \xi} Q_{1}\left(\xi_{0}, \eta_{0}\right)
$$

Substitution of the inverse of eq $13 \mathrm{~A}$ provides the explicit form of $Q_{2}$.

\section{The Case of $2 D$}

In the case of the two dimensions, the situation is far simpler (Figure $3 \mathrm{~b}$ ). The distribution $P_{1}(\theta)$ describing the orientation after the application of the magnetic field to the $z$-direction on the initial random orientation for the period of $t_{1}$ (the first orientation) is given as

$$
P_{1}(\theta)=\frac{\mathrm{e}^{t_{1} / \tau} \sec ^{2} \theta}{2 \pi\left(1+\mathrm{e}^{2 t_{1} / \tau} \tan ^{2} \theta\right)}
$$

For the reorientation (to the $y$-direction), we first rewrite eq $15 \mathrm{~A}$ in terms of $\xi_{0}$ by replacing $\theta$ with $\xi_{0}-\pi / 2$ to obtain $Q_{1}\left(\xi_{0}\right)$. Since $Q_{2}(\xi) \mathrm{d} \xi=Q_{1}\left(\xi_{0}\right) \mathrm{d} \xi_{0}$, we obtain:

$$
\begin{aligned}
Q_{2}(\xi)= & \frac{\mathrm{d} \xi_{0}}{\mathrm{~d} \xi} Q_{1}\left(\xi_{0}\right) \\
= & \frac{\mathrm{e}^{\left(t_{1}+t_{2}\right) / \tau} \csc ^{2}\left(\tan ^{-1}\left(\mathrm{e}^{t_{2} / \tau} \tan \xi\right)\right)}{2 \pi\left(1+\mathrm{e}^{2\left(t_{1}-t_{2}\right) / \tau} \cot ^{2} \xi\right)} \\
& \cdot \frac{\sec ^{2} \xi}{\left(1+\mathrm{e}^{2 t_{2} / \tau} \tan ^{2} \xi\right)}
\end{aligned}
$$

where the inverse of eq $13 \mathrm{~A}$ is used.

\section{REFERENCES}

1. W. J. Jackson, Jr. and H. F. Kuhfuss, J. Polym. Sci., Polym. Chem. Ed., 14, 2043 (1976).

2. W. R. Krigbaum, in "Polymer Liquid Crystals," A. Ciferri, W. R. Krigbaum, and R. B. Meyer, Ed., Academic Press, London, 1982, Capter 10.

3. W. Brostow, in "Physical Properties of Polymers Handbook," J. E. Mark, Ed., American Institute of Physics Press, Woodbury, N.Y., 1996, Chapter 33.

4. G. Maret and A. Blumstein, Mol. Cryst. Liq. Cryst., 88, 295 (1982).

5. F. Hardouin, M. F. Achard, H. Gasparoux, L. Liebert, and L. Strzelecki, J. Polym. Sci., Polym. Phys. Ed., 20, 975 (1982).

6. J. S. Moore and S. I. Stupp, Macromolecules, 20, 282 (1987).

7. A. Anwer and A. H. Windle, Polymer, 32, 103 (1991).

8. E. Ito, H. Sata, and M. Yamato, Mem. Fac. of Tech., Tokyo Metro. Univ., 43, 4677 (1993).

9. S. Kossikhina, T. Kimura, E. Ito, and M. Kawahara, Polym. Eng. Sci., 37, 396 (1997).

10. S. Kossikhina, T. Kimura, E. Ito, and M. Kawahara, Polym. Eng. Sci., in press.

11. T. Shimoda, T. Kimura, and E. Ito, Macromolecules, 30, 5045 (1997).

12. F. Oda, M. Hayashi, S. Nozawa, and I. Shiga, Polym. Prep., Jpn., 37, 2990 (1988).

13. F. Oda, S. Nozawa, M. Hayashi, I. Shiga, M. Kimura, and K. Kajimura, Kobunshi Ronbunshu, 46, 101 (1989).

14. T. Kimura, T. Maeda, H. Sata, M. Yamato, and E. Ito, Polym. J., 27, 247 (1995).

15. A. F. Martins, P. Esnault, and F. Volino, Phys. Rev. Lett., 57, 1745 (1986).

16. A. Anwer and A. H. Windle, Polymer, 34, 3347 (1993).

17. K. Fuhrmann, T. H. Dries, E. W. Fischer, and M. Ballauff, $J$. Polym. Sci., Part B, Polym. Phys., 30, 1199 (1992).

18. N. J. Wagner and L. M. Walker, Macromolecules, 27, 5979 (1994).

19. P. G. de Gennes and J. Prost, in "The Physics of Liquid Crys- 
T. Kimura, H. SATA, and E. Ito

tals," 2nd ed, Clarendon Press, Oxford, 1993.

20. J. Ding and Y. Yang, Polymer, 37, 5301 (1996).

21. J. P. Casquilho, L. N. Goncalves, and A. F. Martins, Liq. Cryst., 21, 651 (1996).

22. P. A. Lebwohl and G. Lasher, Phys. Rev. A, 6, 426 (1972).

23. M. Doi and S. F. Edwards, "The Theory of Polymer Dynamics," Oxford Science Publications, Oxford, 1986, Chapter 3.

24. S. J. Picken, P. Moldenaers, S. Berghmans, and J. Mewis, Macromolecules, 25, 4759 (1992).
25. S. Hanna and A. H. Windle, Polym. Commun., 29, 236 (1988).

26. D. Y. Yoon, N. Masciocchi, L. E. Depero, C. Viney, and W. Parrish, Macromolecules, 23, 1793 (1990).

27. T. Sun, S. K. Bhattacharya, R. W. Lenz, and R. S. Porter, J. Polym. Sci., Polym. Phys. Ed., 28, 1677 (1990).

28. S. Hayase, P. Driscoll, and T. Masuda, Polym. Eng. Sci., 33, 108 (1993).

29. T. Kimura and D. G. Gray, Liq. Cryst., 13, 23 (1993). 